\title{
Effect of NAVA for Dense Optical Communication System
}

\author{
Ghanendra Kumar ${ }^{1}$ and Chakresh Kumar ${ }^{2 *}$ \\ ${ }^{1}$ Department of Electronics and Communication Engineering \\ National Institute of Technology, Delhi-110040, India \\ ${ }^{2}$ University School of Information, Communication \& Technology, \\ Guru Gobind Singh Indraprastha University, New Delhi-110078, India \\ E-mail: *chakreshk@gmail.com
}

\begin{abstract}
Viterbi Algorithm (VA) is basically an algorithm that is implemented for the detection \& estimation of a sequence of symbols in digital communication and signal processing. It calculates a survivor path with minimum metric value, but cannot detect any error. The VA is scrutinized to upgrade the transmission process and provide the plate form in terms of the existence, linear phase noise as well as non-linear phase noise. In contradiction to non-adaptive MLSD (Maximum Likelihood Sequence Detection), the Viterbi algorithm renders the exemplary performance.
\end{abstract}

Keywords: NAVA (Newly Viterbi Algorithm), Optical, EDFA(Erbium-Doped Fiber Amplifier), SOA(Semiconductor Optical Amplifier)

\section{INTRODUCTION}

The need and urgency for optimal performance in optical fiber communication systems has stimulated interest in coherent detection. Many compatible identification (coherent detection) methods are used to support other advanced modulation techniques, for example, M-ary phaseshift-keying (MPSK). The basic principle of PSK modulation is that, for every bit, the phase of the carry signal is changed. The 'M' in the M-ary PSK stands for the degree of the phase shift in the given Phase Shit Keying (PSK) [1-5]. Also, it increases the spectral efficiency by a factor of logbase2 (M). Due to the easy availability of high-level technology \& high speed converters in the form of Analog to digital, the data or the statistics can be preserved on the electric field, and linear impairments such as Chromatic dispersion of the polarization mode, etc. is severly reduced. However, the major issue arises regarding mitigation of carrier phase noise during the usage of local-oscillator, in coherent detection. The presence of phase noise can lead to the reduction of the effective signal-to-noise ratio (SNR) calculated at receiver, decrement the bit error rate (BER) and data rate, corruption of the received signal \& limitation of transmission distance [6-8]. We observe, therefore, that phase noise causes system performance degradation. Several solutions and algorithms have been put forward to eliminate Linear-Phase-Noise (LPN) and Non-LinearPhase-Noise (NLPN) by using either DSP techniques, or with the usage of optical components. Linear phase noise is explored as noise resulting from concise term phase fluctuations in the signal. Fluctuations reveal themselves as a sideband appearing in the form of a noisy spectrum, which is observed to spread out on either sides of the signal. As a result of the reciprocity in the presence of fiber losses in optical medium and the intensified or increased spontaneous-emission (ASE) noise due to wrong setting of higher power amplification. We observe amplified noise and degradation levels in the performance behaviour of modulation techniques that encode data information in the optical carrier phase [9-11]. This is known as non-linear phase noise. The Decision Aided Maximum Likelihood scheme and the Viterbi adaptive maximum likelihood sequence detection schemes, both, are symbol-by-symbol (SBS) algorithms. MLSD's goal is to reduce the application complexity in communication over channels where channels behaviour are nonlinear dispersive. MLSD dramatically diminishes the Viterbi decoder (VD) states and provides a resolution between the two characteristics, the complexity and the performance. In this paper, the performance of MLSD, an Adaptive MLSD, in the existence of linear phase noise \& non-linear phase noise, is studied.

http://doi.org/10.22232/stj.2019.07.01.10 


\section{Effect of NAVA for Dense Optical Communication System}

\section{ADAPTIVE MLSD ALGORITHM}

Primarily, the effect of a multitude of noises are proceeded upon based on a presumption of removal of anomalies like Polarization mode dispersion (PMD) with the help of optical methods and Digital Signal Processor (DSP), and no contribution of the MLSD algorithm is considered for their processing.

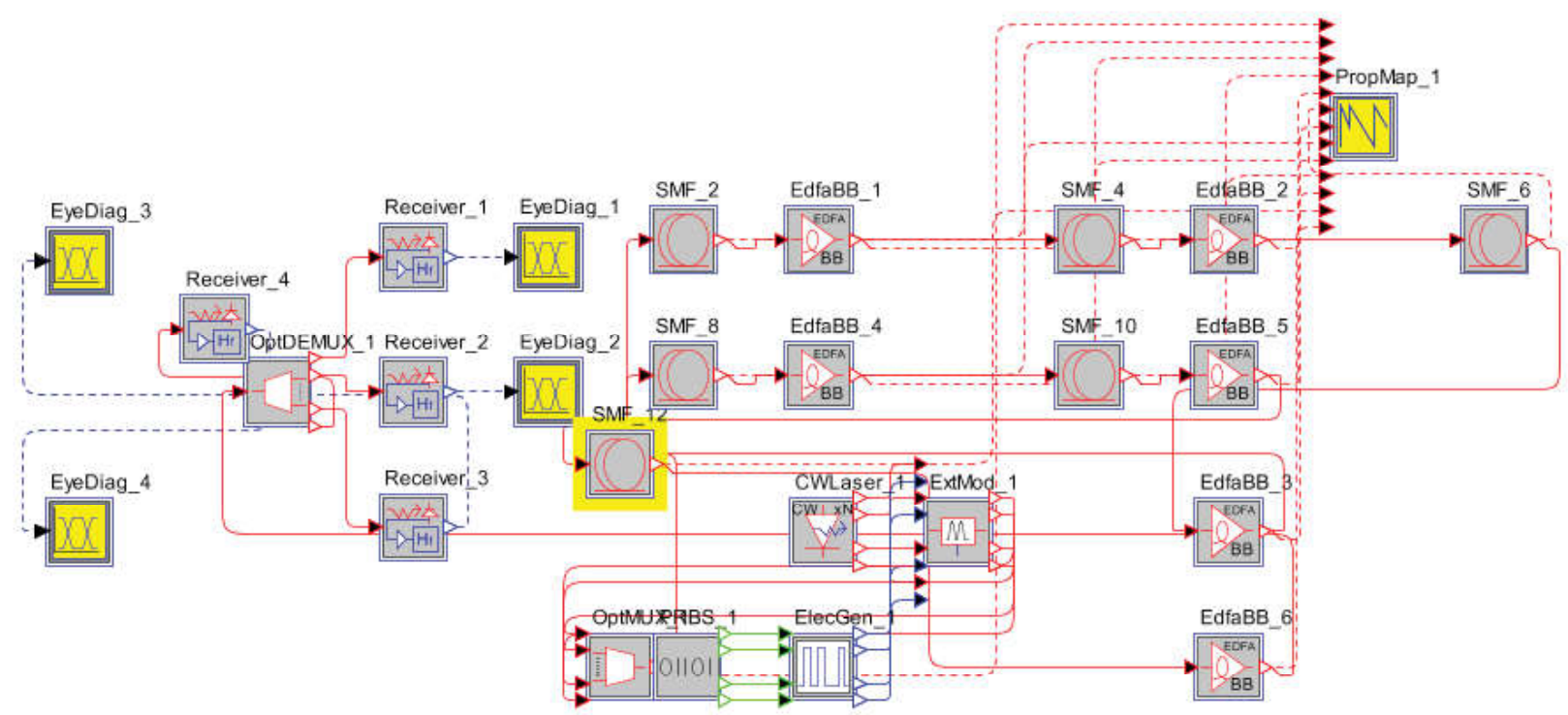

Fig. 1: Proposed Setup for Dense Optical Communication

In the above long haul system given by Fig. 1, optical amplifiers are installed at a range of 100 Kilometers on the fibre span to reduce attenuation in the signal being transmitted. Acquired one made to combine with an LOl signal ( $2 \times 490^{\circ}$ )optical hybrid made up of a ferroelectric material $\mathrm{LiNbO}_{3}$ or Lithium Niobate which has a large acousto-optic and electro-optic coefficients. The output signal later on is further finded by the receiving detectors for the signals. The $\mathrm{i}^{\text {th }}$ symbol quoted as one acquired for the interval (iT, $(\mathrm{i}+1) \mathrm{T})$.

$$
\mathrm{s}(\mathrm{k})=\mathrm{m}(\mathrm{i}) \exp (\mathrm{j} \Theta(\mathrm{i}))+\mathrm{n}(\mathrm{i})
$$

Where $\mathrm{n}(\mathrm{i})$ is sent Mean phase shift keyed or Multi-ary quadrature amplitude modulated singal \& o(i) is AWGN. The wobble of the CPN $\Theta(i)$ is derived from two parts, namely LPN \& NLPN. Priori LPN which is Wiener process $\Theta_{\mathrm{LPN}}(\mathrm{i})=\sum_{\mathrm{h}=-\infty}^{\mathrm{i}} \mathrm{v}(\mathrm{h})$ where $\mathrm{v}(\mathrm{h})$ is tha free and same distributed gaussian random variable having null mean and $\sigma_{\text {LPN }}^{2}=2 \pi \Delta \mathrm{fT}$, with $\mathrm{T}$ denoting duration and $\Delta \mathrm{f}$ denoting the $3 \mathrm{~dB}$ Laser Line width. The later benefactor of PN also known as Non Linear PN which is accumulated by passing it through $\mathrm{N}_{\mathrm{A}}$ is given by -

$\theta_{\text {NL }}(\mathrm{i})=\Sigma^{\mathrm{NA}} \mathrm{i}_{\mathrm{i}-1} \theta_{\mathrm{NL}}(\mathrm{a}, \mathrm{i})=\left.\Sigma^{\mathrm{NA}} \mathrm{i}_{\mathrm{i}-1} \gamma \mathrm{L}_{\mathrm{eff}} \mathrm{E}_{\mathrm{o}}(\mathrm{i}, \mathrm{k})\right|^{2}$
Where the electric field Eo(a,i) $=\mathrm{Eo}(0, \mathrm{i})+\mathrm{N}_{1}(\mathrm{i})+\mathrm{N}_{2}(\mathrm{i})+\ldots$ $+\mathrm{N}_{\mathrm{i}}(\mathrm{i}), \mathrm{N}_{\mathrm{i}}(\mathrm{i})$ being the noise induced by the $\mathrm{i}^{\text {th }}$ amplifier, $\gamma$ is the nonlinearity coeff $\& \mathrm{~L}_{\text {eff }}$ stands for the effective non linear length per fiber span.The viterbi type algorithm is now implemented as the block assessment of the sent signal $m=[m(0)+m(1)+\ldots .+m(D-1)]$ from the acquired signal $r=[r(0)+r(1)+\ldots .+r(D-1)]$ is improbable and won't give us the required output. Therefore, now the sent array is treated as trelli path given in figure 2 and the survivor in each state is the sequence with the max possibility among all the permutations entering that scenario. Hence at an instant $\mathrm{T}=\mathrm{iT}$, the reciever works on the possibility fn $\mathrm{P}(\mathrm{R}(\mathrm{i}) \mid \mathrm{n}(\mathrm{i}))$ of every hypothised subsequence in the sent signal $n(k)=[n(0)+n(1)+n(2)+\ldots . . n(i-1)]$ as per given the acquired sequence $\mathrm{R}=[\mathrm{R}(0)+\mathrm{R}(1)+\ldots \ldots+\mathrm{R}(\mathrm{i}-1)]$. The deciding qty $\mu\left(\mathrm{i}_{\mathrm{o}}, \mathrm{m}\left(\mathrm{i}_{\mathrm{o}}\right)\right)=\mu\left(\mathrm{i}_{\mathrm{o}}-1, \mathrm{n}\left(\mathrm{i}_{\mathrm{o}}-1\right)\right)+\left|\mathrm{r}\left(\mathrm{i}_{\mathrm{o}}\right)-\mathrm{n}\left(\mathrm{i}_{\mathrm{o}}\right) \mathrm{V}_{\mathrm{n}}\left(\mathrm{i}_{\mathrm{o}} \mid \mathrm{i}_{\mathrm{o}}-1\right)\right|^{2}$ only involves linear calculations and it can be subjected to recursive calculations, having it's complexity fixed notwithstanding of array length. In order to apply MLSD we need to calculate a reference phasor from the sequence of previous L symbols.A block length effect arsies in MLSD due to different memory lengths.Therefor we use a better approach called adaptive MLSD algorithm in which we apply a first-order filter for the calculation of reference phasor and it also minimizes theestimation error. Here, sift requires pre state info other 
than $\mathrm{L}$ as the length stored is automatically self-adjusted. So the coherent receiver requires very less memory. The filter is required to be trained to steadty state. This can be done by transmitting a short prologue in the beginning of the sequence. Each data sequence with a length $\mathrm{D}$ is attached with a symbol at the end to cause paths recombination.

\section{DISCUSSION AND RESULTS}

First we will do the analysis of how MLSD will perform in presence of LPN and NLPN wihtout using any adaptation mechanism. For doing so the variance in phase error for reference phasor is derived and it is found that it obeys the Gaussian distribution having a zero mean and variance of MLSD is given by

$$
\begin{gathered}
E[\Delta \theta(k) \mid 2]=\frac{2 L^{2}+3 L+1}{6 L} \sigma^{2}{ }_{L P N} \\
+\left(\frac{1}{L}+1\right) \sigma_{N L}^{2}+\left(\frac{1}{2 L}\right) \sigma_{n^{\prime}}^{2}
\end{gathered}
$$

The resolution between balancing the NLPN, LPN and additive noise is explained by Eq.(5).To minimize error of estimation the optimum value of $L$ can be calculated using Eq.(5) given as

$$
L_{\text {opt }}=\left[\sqrt{\frac{\sigma^{2}{ }_{L P N}+6 \sigma^{2}{ }_{N L}+3 \sigma_{n}^{2} n^{\prime}}{2 \sigma^{2}{ }_{L P N}}}\right]
$$

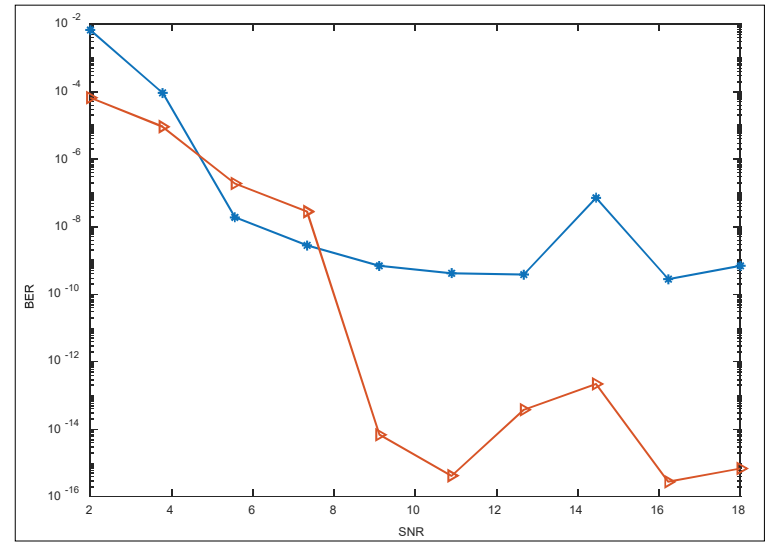

Fig. 2: BER vs. SN Simulation at 25 Gbaud/s QPSK Signal Considering Only LPN with LLW $=200 \mathrm{k} / 5 \mathrm{M}$
Fig. 2 shows a comparison of BER vs. SNR for Adaptive MLSD and MLSD algorithms. It is observed that when the LLW is large, a shorter $\mathrm{L}$ is required by the phase noise for tracking which is varying fast. On the other hand the salient feature of longer $\mathrm{L}$ is that additive noise compensated by it. In case of unknown channel statistics and usage of improper receiver and memory value being employed, it will be observed that especially in the low BER region the performance of BER will degrade.

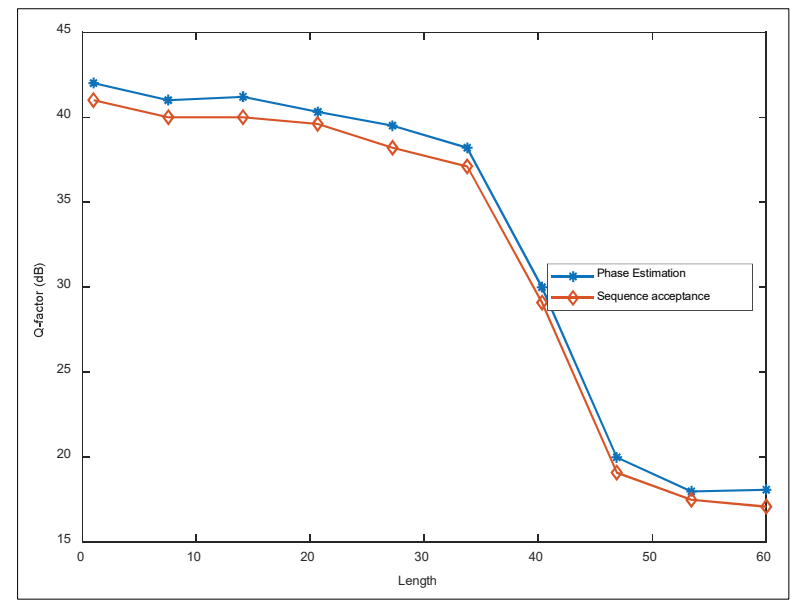

Fig. 3: Curve of Q-factor vs. Memory Length for DAML and MLSD at 25 GBaud/s DQPSK Signals having 20 Spans

Fig. 3 shows a comparison of the plot for Q-factor vs. memory length between MLSD and DAML with only NLPN for a fibre which has 20 spans transmitting in which is a 25 Gbaud/s DQPSK signal. It can be seen that when Q-factor is low sequence detection algorithm(MLSD) excel SBS algorithm(DAML). Increasing the memory length results in the increase in Q-factor for both the algoritms but after certain poitn the curve saturates which is evident from equation (5). Larger the number of amplifiers higher will be the variance of NLPN. So in order to have a higher Q-factor the error variance must be reduced which is possible only by having a longer memory length $\mathrm{L}$. Consequently,the complexity of the receiver is increased which is undesirable. To avoid receiver complexity, we use the adaptive version of MLSD in order to have an optimal performance. The memory requirement for a first-order filter is same as $\mathrm{L}=1$ in MLSD. 


\section{Effect of NAVA for Dense Optical Communication System}

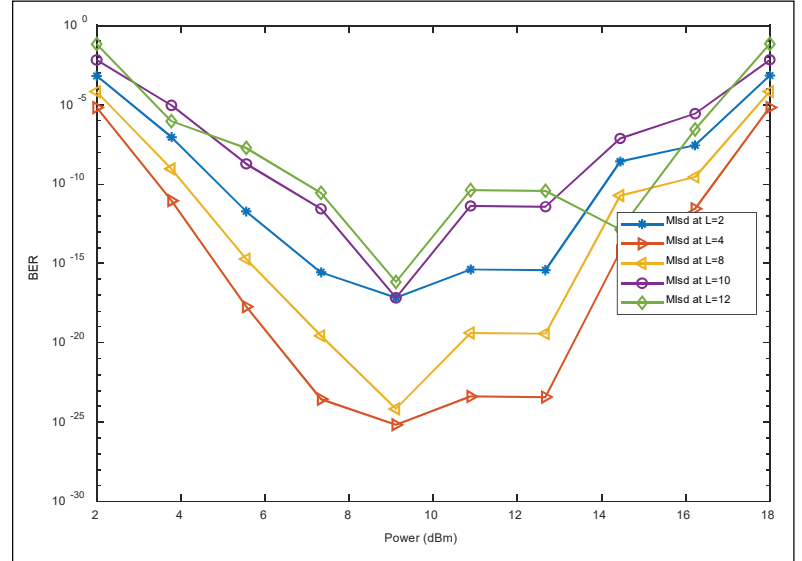

Fig. 4: Curve of BER vs. Launched Power for Adaptive MLSD vs. Non-adaptive MLSD having 23 Spans for LLW $=10 \mathrm{MHz}$ at 25GBaud/s DQPSK

In Fig. 4, we consider both type of noises i.e. LPN and NLPN which is a real situation. It is found that simply increasing memory length due to LPN does not made any improvement in the error-rate performance. In the region where SNR is low,with increasing SNR the BER decreases as the dominant distortion in this region is additive noise. In the region where the launched power is greater than optimum launched power the noise is mainly because of Non-Linear Phase Noise which is due to the fact that power of signal is large. It can be seen from Fig. 5 that at optimum launched power there is a considerable improvement in BER performance of adaptive MLSD over non-adaptive MLSD which can be even more better if receiver knows the statistics of the channel.

\section{CONCLUSION}

Adaptive MLSD algorith of the type Viterbi for optical systems which are coherent in nature having NLPN \& LPN both was studied and it was concluded that favourable and best performance was delivered with the complexity of the receiver being low.

\section{REFERENCES}

Sorokin E, Naumov S, Sorokina I T (2005) Ultra broadband infrared solid-state lasers: IEEE J. Sel. Topics Quantum Electron 11: 690-712.

Lo C Y, Huang K Y, Chen J C, Tu S Y, Huang S L (2004) Glass-clad Cr4p: YAG crystal fiber for the generation of super wideband amplified spontaneous emission: Opt. Lett. 29: 439-441.

Alcock A J (2005) Ultrashort pulse Cr4p: YAG laser for high precision infrared frequency interval measurements: Opt. Exp.13: 8837-8844.

Tang P (2013) Topological insulator: Bi2Te3 saturable absorber for the passive Q-switching operation of an in-band pumped 1645-nm Er:YAG ceramic laser: IEEE Photon. J. 5: 1500707.

Zhang X (2014) Widely tunable, narrow bandwidth polycrystalline ceramic Er: YAG laser with a volume Bragg grating: Opt. Exp. 22: 7154-7159.

Liu J (2013) Volume Bragg grating-based tunable Er,Yb fiber lasers covering the whole C- and L- band: IEEE Photon. Technol. Lett. 25: 1488-1491.

Wu B, Liu Y, Dai Z, Liu S (2007) Stable narrow linewidth Er-doped fiber laser at $1550 \mathrm{~nm}$ : Microwave: Opt. Technol. Lett. 49: 1453-1456.

Desmoulins S, Teodoro F D (2007) Watt-level, high-repetition-rate, mid-infrared pulses generated by wavelength conversion of an eye-safe fiber source: Opt. Lett. 32: 56-58.

Wang M, Zhu L, Chen W, Fan D (2012) Efficient all-solid-state midinfrared optical parametric oscillator based on resonantly pumped 1.645 m Er:YAG laser: Opt. Lett. 37: 2682-2684.

Philippov V (2004) High-energy in-fiber pulse amplification for coherent lidar applications: Opt. Lett. 29: 2590-2592.

Ma J (2009) Experimental investigation of radiation effect on erbium-ytterbium co-doped fiber amplifier for space optical communication in low-dose radiation environment: Opt. Exp.17: 15 571-15 577. 